\title{
Ende und Erbe der lateinisch-heidnischen Geschichtsschreibung
}

\author{
Giuseppe Zecchini
}

Die heidnische lateinische Geschichtsschreibung neigt zwischen dem 4. und dem 5. Jahrhundert ihrem Ende zu, und zwar mit einer Textproduktion - den Annales von Nicomachus Flavianus, die um 389/391 dem Theodosius gewidmet wurden; der Epitome de Caesaribus, die zum großen Teil daraus abgeleitet ist und in den ersten Jahren des 5. Jahrhunderts verfasst wurde; dem biografischen corpus der Historia Augusta, das im Grunde im letzten Jahrzehnt des 4. Jahrhunderts vollendet war, aber wahrscheinlich in den ersten Jahrzehnten des 5. Jahrhunderts auf den neusten Stand gebracht wurde ${ }^{1}-$ einer Textproduktion also, die auf ein einziges politisch-kulturelles Milieu zurückzuführen ist, das Milieu der bedeutenden Senatorenfamilien der Nicomachi und der Symmachi.

Ein Jahrhundert später, als die antike Kultur in Italien zur Zeit Theoderichs zum letzten Mal aufblühte, finden wir im Mittelpunkt der historiografischen Produktion einen Symmachus und einen Gelehrten, Cassiodor, der seiner Verwandtschaft mit den Anicii Geltung verschaffen wollte. ${ }^{2}$ Selbstverständlich sind sowohl Q. Aurelius Memmius Symmachus als auch Cassiodor schon Christen: Ihre ausgiebige historiografische Produktion (die Historia Romana des Ersten, das Chronicon, die Historia Gothorum und die Historia Tripertita des Zweiten) besteht aber aus einer Mischung der historiografischen Gattungen der neuen christlichen, und jetzt schon frühmittelalterlichen Kultur wie der Chronologie (die im Grunde auf den Eusebius-Hieronymus zurückzuführen ist) und der Kirchengeschichte, und der Gattungen der alten heidnischen Kultur wie der römischen Geschichte und der ethnografischen Monografie.

1 Über diese Gruppe von Texten vgl. im allgemeinen G. Zecchini, Ricerche di storiografia latina tardoantica, Roma 1993, 39-64; die neueste Hypothese über die Entstehung der Historia Augusta stammt von M. Festy, Les Nicomaques auteurs de l'Histoire Auguste. La jalousie des méchants, in: Compte-rendus de l'Académie des Inscriptions et Belles Lettres 2004, 757-767 ; Id., L'Histoire Auguste et les Nicomaques, in: G. Bonamente-H. Brandt (Hrsgg.), Historiae Augustae Colloquium Bambergense, Bari 2007, 183-195.

2 Cassiod. Ordo gener. Cassiod. und Instit. I,23,1. 
Um genauer zu sein, kann die Historia Romana in 7 Büchern, aus denen mehr als die Hälfte dem Reich gewidmet ist (das fünfte Buch behandelte bekanntlich Maximinus Thrax), vielleicht auch mit den Historiae adversus paganos des christlichen Orosius in Verbindung gebracht werden, die auch aus 7 Büchern bestehen; sie lehnt sich aber eher an die Annales ihres heidnischen Vorgängers an (der aber 431 rehabilitiert wurde). ${ }^{3}$ Nicht nur sind die Annales auch in 7 Bücher eingeteilt, sie stimmen vielmehr - und das ist wichtiger - in den Inhalten überein: Es handelt sich um eine römische Geschichte, und nicht um eine Universalgeschichte wie die des Orosius, der die ganze Reichsgeschichte im siebten und letzten Buch komprimierte. Es geht um eine Geschichte, die von einem Christen verfasst wurde, aber einen traditionellen Textbauplan aufweist, der auf die vorhergehende heidnische Geschichtsschreibung zurückgreift.

Die Historia Gothorum (d. h. De origine actuque oder actibusque Getarum) in 12 Büchern musste ihrerseits nicht nur die gotische mündliche Tradition hinter sich haben, sondern auch auf der einen Seite einen „Klassiker“ der antiken Chorografie wie Mela und zwei Universalgeschichten, die heidnische des Pompeius Trogus (eher diese als das entsprechende, von Justinus zusammengestellte Kompendium) und die christliche des Orosius, ${ }^{4}$ auf der anderen Seite die griechische ethnografische Tradition. Diese hatte auch Klassiker der lateinischen Geschichtsschreibung inspiriert wie die Origines von Cato, die gerade von Cassiodor im Ordo generis Cassiodororum ${ }^{5}$ erwähnt werden, die Germania (d.h. De origine et situ Germanorum) des Tacitus und die anonyme Origo gentis Romanae, die mit dem De viris inlustribus und mit Aurelius Victor in das Corpus tripertitum gesammelt wurde; insbesondere hatte sie nicht wenige Getiká und/oder Skythiká hervorgerufen: Auch wenn diese Texte, von Dion Chrysostomos bis Dexippos und Ablabios, von Cassiodor weder gelesen noch nachgeschlagen wurden, ${ }^{6}$ bedeutet das nicht unbedingt, dass ihm die gesamte griechisch-lateinische Gattung der Literatur über die origo und die mores der germanischen Völker unbekannt war: er fügte sich bewusst in diese Tradition ein.

Meiner Meinung nach war Jordanes, ein gotischer aber mit den Anicii in Verbindung stehender Geistlicher, um 551/552 in Konstantinopel sowohl auf die Historia Romana von Symmachus als auch auf die Historia Gothorum von Cassiodor gestoßen und hatte sie für seine Romana (d.h. De origine actibusque gentis Romanorum) bzw. Getica (d.h. De origine actibusque Getarum) wiederaufgenommen. Während der Einfluss von Cassiodor allgemein anerkannt wird,

3 CIL VI,1783 = ILS 2948.

4 Vgl. Zecchini (s. Anm. 1), 203 und 209.

5 Ll. 6-7: Symmachus... qui antiqui Catonis fuit nouellus imitator.

6 Vgl. wieder Zecchini (s. Anm. 1), 209. 
hat Croke, und nach ihm Heather, den Einfluss von Symmachus abgelehnt; ${ }^{7}$ Callu und ich haben dagegen neue und überzeugende Gründe zur partiellen Unterstützung der alten These von Enßlin angeführt, die die Romana von Jordanes als einen Abriss der Historia Romana des Symmachus betrachtete. ${ }^{8}$ Eigentlich sollte man nicht von Abriss reden, so wie die Getica kein Abriss der Historia Gothorum von Cassiodor sind, aber die nicht gerade spärlichen „westlichen“ Materialien, die in den Romana vorhanden sind, können nicht von chronologischen byzantinischen Quellen herkommen, wie Croke hingegen meint. Ihre Ableitung aus Symmachus bleibt die am meisten ökonomische und daher zu bevorzugende Hypothese.

Tatsache ist, dass sich in den zwei relativ knappen und gut lesbaren Monografien von Jordanes Formen und Eigenschaften kristallisieren, die zum Teil aus der klassischen Antike stammen; dadurch werden sie dem frühen Mittelalter, wo beide historiografischen Texte weit verbreitet sind, überliefert, wenngleich durch die Vermittlung von schon christlichen Autoren wie Symmachus und Cassiodor und durch eine weitere Verarbeitung des Jordanes selbst.

Das berühmte Zitat aus der Vita Maximini der HA in der Historia Romana von Symmachus und von hier in den Getica von Jordanes ${ }^{9}$ bezeugt das andauernde Interesse für Kaiserbiografien im Rahmen der Familientradition der Symmachi; auch die Untersuchung der handschriftlichen Überlieferung weist auf das Vorhandensein von Abschriften der $H A$ in der Privatbibliothek der Symmachi in Rom und in der Biblioteca Capitolare in Verona, einer der Hauptstädte des ostgotischen Reiches $;^{10}$ die Epitome de Caesaribus selbst, die aus demselben Kreis hervorkam, zeigte übrigens schon eine nach Kaiserbiografien strukturierte Darstellung der Reichsgeschichte, die also als eine Kaisergeschichte aufgefasst wurde. Auf den Kreis der Anicii, die um 540 in Konstantinopel im Exil waren, könnte uns auch der Anonymus Valesianus II zurückführen, laut einer wohl beeindruckenden, aber nicht von allen geteilten Hypothese: ${ }^{11}$ Es ist ein merkwürdiger Text, mit einem einzigen Verfasser aber

7 B. Croke, A.D. 476: The Manufacture of a Turning Point, Chiron 13, 1983, 81-119 (wiederaufgenommen in Id., Latin Historiography and the Barbarian Kingdoms, in: G.Marasco [Hrsg.], Greek and Roman Historiography in Late Antiquity, LeidenBoston 2003, 349-389, 372); P. Heather, The Historical Culture of Ostrogothic Italy, in: Teoderico il Grande e i Goti d'Italia, Spoleto 1993, I, 317-353, 332.

8 J. P. Callu, La première diffusion de l'Histoire Auguste, in: BHAC 1982/83, Bonn 1985, 89-129 = Id., Culture profane et critique des sources de l'antiquité tardive, Rome 2006, 159-199; Zecchini (s. Anm. 1), 73-83.

9 Iordan. Get. XV,83-88.

10 Zecchini (s. Anm. 1), 47-49.

11 G. Zecchini, L'Anonimo Valesiano II: genere storiografico e contesto politico, in: Teoderico il Grande e i Goti d'Italia, Spoleto 1993, II, 809-818; Croke (s. Anm. 7), 
nach historiografischen Gattungen dreigeteilt. Er besteht nämlich aus einem ersten Teil, der wie eine Kaisergeschichte aufgebaut ist, aus einem zweiten, dem mittleren Teil, der offensichtlich suetonischer Abstammung ist (im $\ 62$ ist eine Anekdote wortwörtlich aus dem Divus Claudius 15,2 abgeschrieben), und aus einem letzten Teil, der sich an die Heiligengeschichte orientiert (das Zitat aus der Vita S. Severini von Eugippius in den Abschnitten 46-48 kündigt das unmissverständlich an). Hier möchte ich vor allem den Einfluss von Sueton als biografischer Vorlage hervorheben, die gleiche Vorlage der $H A$, die dieses Mal von einem christlichen Autor und für einen christlichen Kaiser, den arianischen Theoderich, wieder aufgenommen wird, aber immer innerhalb der gleichen senatorischen Kulturtradition.

Suetons Muster ist hier übrigens zum letzten Mal bezeugt, und verschwindet dann für etwa drei Jahrhunderte, bevor es in der karolingischen Renaissance dank der Vita Karoli von Einhard wieder auftaucht. Noch strenger ist das Schicksal mit Marius Maximus verfahren, der von den römischen Senatoren zur Zeit des Ammianus Marcellinus, also etwa am Ende des 4. Jahrhunderts, noch gelesen und geschätzt wurde, ${ }^{12}$ aber gleich danach endgültig in Vergessenheit geriet. Was die $H A$ anbelangt, hat sie Paulus Diaconus wahrscheinlich gekannt und für seine Historia Langobardorum ${ }^{13}$ benutzt, das wäre aber die einzige Ausnahme zwischen der Mitte des 6. und dem 9. Jahrhundert, als dieses biografische Sammelwerk dann wieder in Umlauf kam. ${ }^{14}$

Abgesehen von der biografischen Tradition senatorischer Prägung möchte ich den großen spätantiken Erfolg von Valerius Maximus erwähnen, der von den Epitomen des Iulius Paris und des Ianuarius Nepotianus bezeugt ist, beide aus dem 4. Jahrhundert; die erste wurde zur Zeit Theoderichs von einem gewissen Domnulus abgeschrieben, wie der Unterschrift des zehnten Buches zu entnehmen ist. ${ }^{15}$ Valerius Maximus ist kein Biograf, aber die an Anekdoten

357, lehnt die Herkunft des Textes aus dem Kreis der Anicii in Konstantinopel ab; seiner Meinung nach sollte er eher ein Fragment aus den Chronica von Maximian, von 546 bis 553 Bischof von Ravenna, sein.

12 Amm. XXVIII,4,14.

13 So die neue Hypothese von A. A. Settia, Aureliano imperatore e il cavallo di re Alboino. Tradizione ed elaborazione nelle fonti pavesi di Paolo Diacono, in: P. Chiesa (Hrsg.), Paolo Diacono, Udine 2000, 487-504; ich werde sie demnächst a.a.O. behandeln.

14 Callu (Diffusion, s. Anm. 8) und Id.-O. Desbordes-C. Bertrand, L'Histoire Auguste et l'historiographie médiévale, Revue d'histoire des texts 14/15, 1984/85, 97-130= Callu (Culture, s. Anm. 8), 201-234, sowie O. Pecere, Il codice palatino dell'Historia Augusta come edizione continua, in: Id.-M. D. Reeve (Hrsgg.), Formative Stages of Classical Traditions: Latin Texts from Antiquity to the Renaissance, Spoleto 1995, 323-369.

15 J. R.Martindale, PLRE II, Cambridge 1980, 374-375. Zur späteren Rezeption von Iulius Paris vgl. G. Billanovich, Il Petrarca e gli storici latini, in: G. Bernardoni Trezzini (Hrsg.), Tra latino e volgare. Per Carlo Dionisotti, Padova 1974, I, 67-145. 
sehr reichen Materialien seines Werkes lieferten der biografischen Neugierde ausgiebigen Stoff.

Nicht besonders auffällig scheint hingegen das Vorhandensein eines autobiografischen Textes wie Caesars Commentarii beim Patrizier Parthenios in Ravenna, ${ }^{16}$ immer zu Theoderichs Zeit: Es ist tatsächlich rein zufällig und der gallo-römischen Herkunft des Parthenios zuzuschreiben, da Caesar keine Rolle im kulturellen Erbe des ostgotischen Italiens spielte und auf jeden Fall keinen Einfluss auf die mittelalterliche Geschichtsschreibung ausübte. Insgesamt sind die Biografen der neuen Epoche fast ausschließlich an der hagiografischen Gattung der Vitae Sanctorum interessiert, und als Paulus Diaconus tatsächlich die neue Gattung der gesta episcoporum mit dem Liber de episcopis Mettensibus schuf, war der Liber pontificalis seine relevanteste Vorlage: ${ }^{17}$ Die christliche Biografie scheint, in allen ihren Formen, den heidnischen Vorgängern nichts schuldig zu sein.

Wenn wir jetzt unsere Analyse jenseits der wichtigen, aber engen Grenzen der römischen Aristokratie erweitern, begegnet uns eine Gruppe von heidnischen Texten, die theoretisch wegen ihrer Funktionalität einen tiefen Einfluss auf die historische Kultur des Mittelalters hätten ausüben können: Ich meine Justinus, Eutrop (in beschränktem Maße auch Festus und Florus), und das Corpus tripertitum. ${ }^{18}$

Justinus lieferte ein Kompendium der Universalgeschichte, das vom christlichen Orosius nicht ersetzt werden konnte, weil dieser stilistisch wesentlich komplizierter war; Orosius selbst und vielleicht noch Cassiodor mit der Historia Gothorum hatten Zugang zu den Historiae Philippicae des Pompeius Trogus; diese waren aber höchstwahrscheinlich schon im 4. Jahrhundert und

16 Arator Ad Parthenium 39-40; vgl. O. Pecere, La cultura greco-romana in età gota tra adattamento e trasformazione, in: Teoderico il Grande e i Goti d'Italia, Spoleto 1993, I, 355-394, 367, und auch Heather (s. Anm. 7), 320. Über die Unterschriften in Caesars Text vgl. auch O. Pecere, La tradizione dei testi latini tra IV e V secolo attraverso i libri sottoscritti, in: A. Giardina (Hrsg.), Società romana e impero tardoantico, IV, Roma-Bari 1986, 19-81.

17 W. Goffart, Paul the Deacon's „Gesta episcoporum Mettensium“ and the early Design of Charlemagne's Succession, Traditio 1986, 59-84; M. Sot, Le Liber de episcopis Mettensibus dans l'histoire du genre „Gesta episcoporum“, in: Chiesa (s. Anm. 13), $527-550$.

18 Selbstverständlich zählt Hegesippos zu den antiken Geschichtsschreibern, die im frühen Mittelalter meistgelesen und weit verbreitet waren. Ich behandle ihn nicht, weil es sich im Grunde um die lateinische Fassung von Flavius Josephus handelt (J. P. Callu, Le „De bello Iudaico“ du Pseudo-Hégésippe: essai de datation, in: BHAC 1984/85, Bonn 1987, 117-142), einem weder lateinischen noch heidnischen, wohl aber jüdischhellenistischen Historiker. 
gerade von Justinus zusammengefasst worden, ${ }^{19}$ und in der Zeit nach Cassiodor trat die gekürzte Fassung eher in den Vordergrund, anstelle des langen und komplizierten Originals. Aus dem unvergleichlichen Reichtum seiner handschriftlichen Überlieferung ${ }^{20}$ können wir schließen, dass Justinus der meist gelesene, meist abgeschriebene und meist verbreitete heidnische Historiker des frühen Mittelalters war, aber ein entsprechender Einfluss auf die historiografische Produktion jener Jahrhunderte ist nicht festzustellen. Wir befinden uns also vor einem Autor, der wohl bekannt war, aber nicht Schule machte: Man muss bis auf die Zeit des Kaisers Heinrich II. warten, bis wir Berichte haben, die vom Bedürfnis bezeugen, in der Hofbibliothek eine handschriftliche Anthologie ${ }^{21}$ historischer Texte zu besitzen, u.a. der sogenannten Exordia Scythica aus dem Justinus; aber auch diese Angabe spricht nicht unbedingt, um ehrlich zu sein, für irgendeinen historiografischen Einfluss.

Was Eutrop, Festus und Florus - der letzte schon von Orosius als Quelle benutzt - anbelangt, kann man eine mögliche Kenntnis vermuten, da diese Texte kurz und einfach sind, da sie sich erhalten haben und Abrisse der römischen Geschichte lieferten, des interessantesten und bedeutendsten Teils der Universalgeschichte, der weiterer Untersuchung verdiente. Unmittelbare und massive Benutzung von Eutrop allein ist aber nur bei Paulus Diaconus belegt, aber auch in diesem Fall müssen wir die Benutzung einer Quelle von der Übernahme eines historiografischen Musters unterscheiden.

Die Geschichtsschreibung der langobardischen Zeit vor der bedeutenden Persönlichkeit von Paulus Diaconus ist durch zwei kleinere Werke vertreten, die aber für unser Thema von größtem Interesse sind: die verloren gegangene Historia de gestis Langobardorum des Secundus von Trient und die Origo gentis Langobardorum, die dem edictum Rotharii vorangestellt ist. ${ }^{22}$

19 Trogus und Orosius, Trogus und Cassiodor: Vgl. Zecchini (s. Anm. 1), 157 bzw. 204. Zur Datierung der Epitome von Justinus: R. Syme, The Date of Justin and the Discovery of Trogus, Historia 37, 1988, 358-371 = Roman Papers, VI, Oxford 1991, 358-371.

20 Ausreichend sei der Hinweis auf das klassische Werk von M. Manitius, Handschriften antiker Autoren in mittelalterlichen Bibliothekskatalogen, Leipzig 1935.

21 Die Bambergensis Hist. 3; darüber jetzt P. Chiesa, Storia romana e libri di storia romana fra IX e XI secolo, in: Roma antica nel Medioevo, Milano 2001, 231-258, 252-254.

22 Für eine Einführung in beide Werke verweise ich auf die neulich erschienene, grundlegende Studie von W. Pohl, Memory, Identity and Power in Lombard Italy, in: Y. Hen-M. Innes (Hrsg.), The Uses of the Past in the early Middle Ages, Cambridge 2000, 9-28 (insbes. S.18 über die wichtige Beziehung von Secundus von Trient und der Königin Theodelinda: Er war ihr geistlicher Vater und taufte um 600 ihren Sohn, den künftigen König Adalohald). Über Paulus Diaconus und die Historia Langobardorum vgl. auch W. Pohl, Paulus Diaconus und die Historia Langobardorum: Text und Tradition, in: Historiographie im frühen Mittelalter, Wien 1994, 375-405; Id., Paolo 
Der erste Text geht auf den Anfang des 7. Jahrhunderts zurück, weil Secundus 612 starb; der zweite ist um 668/9, also ein Jahrhundert vor der Monografie von Paulus, entstanden, die um 786/7 zu datieren ist. Die Historia von Secundus ist nicht einfach als Chronik zu bezeichnen, denn sie strebte, trotz ihrer Kürze (succinctam de Langobardorum gestis... historiolam - so beschreibt sie Paulus selbst), ${ }^{23}$ nach etwas Höherem. Die Aufmerksamkeit, die sowohl den Wetterzuständen als auch den Ereignissen des merowingischen Reiches geschenkt wird, verrät eigentlich den Einfluss der Libri historiarum des Gregor von Tours ${ }^{24}$ des einzigen wichtigen historiografischen Werkes der Zeit, das 591, also etwa zwanzig Jahre vorher, vollendet wurde. Dass im Titel die Wendung de Langobardorum gestis beibehalten wurde (statt actibus wie bei Jordanes), deutet vielleicht auf die Res gestae von Ammianus Marcellinus. Diese wurden wahrscheinlich von Paulus Diaconus benutzt, und es ist daher schwer festzulegen, ob die Wendung eigentlich von Secundus stammt oder nicht; das Wort historia im Titel ist wohl mit Gregor von Tours, aber auch mit der italischen Tradition zu verbinden, die auf die Historiker der ostgotischen Zeit zurückgeht.

Diese historia beschränkt sich auf die res gestae der Langobarden und bewirkt dadurch die Eingrenzung der historischen Perspektive auf ein regionales Gebiet; sie stillt aber das Bedürfnis nach einem zeitgenössischen Zeugnis aus erster Hand, und das entspricht dem echten Wert des Wortes historia der antiken historiografischen Tradition. Die Verkleinerungsform historiola sollte schließlich nicht dem Paulus zugeschrieben werden, was ihr sonst eine fast abwertende Färbung verleihen würde, sondern dem Secundus selbst - und in diesem Fall erweckt sie den Eindruck bewundernswerter Bescheidenheit. Das Wort historiola ist dann Widerhall des Wortes storiuncola, das Jordanes für seine Romana zwei Menschenalter vorher benutzt hatte ${ }^{25}$ und bezeugt, dass sich die Historiker zwischen Spätantike und frühem Mittelalter dessen bewusst waren, selbst wenn unwürdig, die Erben der bemerkenswerten Tradition der historiae zu sein, der gotischen von Cassiodor, der römischen von Symmachus, der universellen von Orosius.

Auf jeden Fall stellt Secundus von Trient für uns das einzige Glied einer historiografischen Kette dar, die in Italien die Autoren der klassischen Epoche

Diacono e la costruzione dell'identità longobarda, in: Chiesa (s. Anm. 13), 413-426; D. Bullough, Ethnic History and the Carolingians. An alternative Reading of Paul the Deacon's Historia Langobardorum, in: Chr. Holdsworth (Hrsg.), The Inheritance of Historiography 350-900, Exeter 1986, 85-105.

23 Paul. HL IV,40; Secundus von Trient wird auch in HL III,29 und IV,27 von Paulus zitiert.

24 So K. Gardiner, Paul the Deacon and Secundus of Trent, in: B. Croke-A. M. Emmett (Hrsgg.), History and Historians in Late Antiquity, Oxford 1983, 147-154.

25 Iordan. Rom. 6. 
Theoderichs mit Paulus Diaconus und dem Wiederaufblühen der Geschichtsschreibung in der karolingischen Zeit verbindet.

Die spätere Origo wurde neulich sogar mit dem ersten Teil des Corpus tripertitum verglichen, ${ }^{26}$ d.h. mit der Origo gentis Romanae, aber ohne Grund: Dieses letzte Werk ist das Ergebnis antiquarischer Gelehrsamkeit, reich an Zitaten (oder Pseudo-Zitaten) aus antiken und seltenen Autoren $;{ }^{27}$ die Origo gentis Langobardorum ist hingegen ein kurzer Text, worin ein Bericht in Gesprächsform über die „mythische“ Vergangenheit der Langobarden (vor der Völkerwanderung) und die Reihenfolge der Könige miteinander verwoben sind; das Ganze wurde mit spärlichen, chronikartigen Angaben bereichert. Wir haben es mit jener Literatur über die origines der germanischen Völker zu tun, die allmählich zur Geschichte wurde, indem die Zeit der Wanderung sich in die Zeit der ständigen Ansiedlung verwandelte und indem man mit Rom oder mit anderen stabilen und starken Mächten (der Kirche, dem Frankenreich) in Kontakt kam. Cassiodor hatte die wirksamste Formulierung für diesen Prozess verfasst, wo er schrieb: originem Gothicam historiam fecit esse Romanam. Dieser Übergang von origo zu historia ist das sprachliche $\mathrm{Ab}-$ schlusszeugnis einer historiografischen Entwicklung, die die vorausgehende, politische Entwicklung mit ausgezeichneter Klarheit zur Sprache bringt. ${ }^{28}$ Selbstverständlich konnte sich die origo Langobardica nicht mehr in eine historia Romana verwandeln, wohl aber in eine historia, und das bewirkte Paulus. Was aber in diesem Zusammenhang wichtig ist, ist es eben, den Einfluss vom ersten Teil des Corpus tripertitum aus der Entstehungsgeschichte der Origo gentis Langobardorum auszuschließen: Diese muss wiederum auf Cassiodor ,,über Jordanes" zurückgeführt werden; das Corpus tripertitum hingegen, sowie Eutrop, Festus und Florus überleben die frühmittelalterlichen Jahrhunderte, sie sind jedoch historiografisch nicht wirksam.

Paulus Diaconus verfasste die Historia Romana in 16 Büchern um 770; neben seinen zwei Hauptquellen, eben Eutrop und Orosius, zieht er zahlreiche Nebenquellen (Jordanes, das Chronicon des Prosper von Aquitanien, die Epitome, gelegentlich auch Materialien aus Hieronymus, Frontinus und Soli-

26 Aus L. Capo, Paolo Diacono e il mondo franco: l'incontro di due esperienze storiografiche, in: Chiesa (s. Anm. 13), 39-74, 71 Anm. 45.

27 Man vergleiche darüber die kommentierte Ausgabe von G. D'Anna, Origine del popolo romano, Milano-Verona 1992.

28 Cassiod. Variae IX,25,5 nach der Auslegung von Zecchini (s. Anm. 1), 194-195; anders P. Heather, Goths and Romans 332-489, Oxford 1991, 53, und ihm folgt Croke (s. Anm. 7), 363. Er deutet diese Bezeichnung rein literarisch, als Verschmelzung gotischer Traditionen und antiker Materialien; Heather (s. Anm. 7) selbst hat noch überzeugender hervorgehoben, dass die Historia Gothorum sich gut in das politisch-kulturelle Programm einfügt, das von Theoderich gefördert wurde und auf der Romanitas beruhte. 
nus) ${ }^{29}$ das Thema des Werkes und die entsprechende Überschrift nehmen Abstand sowohl von Eutrop, der die römische Geschichte behandelte, aber ein Breviarium verfasste, als auch von Orosius, der Historiae schrieb, sich aber mit der Universalgeschichte beschäftigte. Die einzige schon existierende Historia Romana war die des Symmachus, die Paulus dank des berühmten Zitats in den Getica von Jordanes kannte; übrigens unterbricht Paulus sein Werk im Jahr 552 abrupt, nicht so sehr weil dieses Datum für den langobardischen Historiker eine epochale Bedeutung besaß, sondern, wie zutreffend schon bemerkt wurde, ${ }^{30}$ eher wegen der rein technischen Tatsache, dass Jordanes nur bis dahin kam und danach standen Paulus keine Quellen mehr zur Verfügung: Nach Jordanes begann, in der Mitte des 6. Jahrhunderts, eine historiografische Nacht, die man nicht zu beleuchten wusste. Jordanes spielte eine wichtige Rolle, nicht nur weil er der letzte, dem Paulus zur Verfügung stehende Autor ist, sondern auch weil er ihm das Modell für seine Tätigkeit als Historiker durchaus lieferte. Wenn wir nämlich beide großen Werke von Paulus miteinander vergleichen, die Historia Romana und die Historia Langobardorum, kommt die Ähnlichkeit mit dem entsprechenden Paar des Jordanes, Romana und Getica, deutlich und unleugbar zum Vorschein: Bei beiden Autoren findet man eine ähnliche römische Geschichte und die ethnografische Monografie über das eigene Volk (die Goten, die Langobarden), das dazu bestimmt war, in die höhere Einheit des römischen Reiches Justinians im ersten, des Heiligen Römischen Reiches der Franken im anderen Fall einzufließen und sich darin aufzulösen.

Die antiken Muster werden also wieder durch die christliche Vermittlung von Jordanes, die ich schon erläutert habe, auch an Paulus weitergeleitet; von sich aus fügt Paulus einen weitschweifigen erzählerischen Diskurs hinzu und demzufolge eine Einteilung in zahlreiche Bücher, die bei Jordanes nicht zu finden ist. Paulus strebt offensichtlich danach, mit Orosius $\mathrm{zu}$ wetteifern, vielleicht sogar mit den Werken eines Symmachus oder eines Cassiodor - auf jeden Fall, wie wir sehen werden, mit dem ihm näher stehenden Gregor von Tours; er strebt aber bestimmt danach, der Geschichtsschreibung wieder zur literarischen Würde zu verhelfen, einer Würde, die nach der Zeit der ostgotischen Klassiker verloren gegangen war.

Die bisher durchgefuihrte Analyse beschränkte sich darauf, die Entwicklung der Geschichtsschreibung zu verfolgen von der noch heidnischen Produktion der Spätantike über die Epoche Theoderichs bis zu den ersten Anfängen der karolingischen Renaissance, und das nur im italischen Bereich. Wenn unsere Untersuchung auch die ehemalige Provinz-Welt der anderen

29 Eine Auflistung der Quellen in Chiesa (s. Anm. 21), 241.

30 Aus L. B. Mortensen, Impero romano, Historia Romana e Historia Langobardorum, in: Chiesa (s. Anm. 13), 355-366. 
römisch-germanischen Reiche miteinbeziehen würde, würden wir feststellen, dass die spätantike Geschichtsschreibung im vandalischen Afrika reichlich blühte; sie stellt aber eine eigenständige und in sich geschlossene Erscheinung dar, von Orosius bis Fulgentius, ohne heidnische Vorgänger und ohne jegliches mittelalterliches Erbe in einer Welt, die bald den Arabern zum Opfer fallen würde. ${ }^{31} \mathrm{Im}$ westgotischen Spanien hat die reiche Chronikproduktion keinen heidnischen Hintergrund, und nur für die Historia Gothorum, Wandalorum et Sueborum des Isidor von Sevilla kann man einen Einfluss der Literatur über die origines (Tacitus' Germania?), insbesondere der Historia Gothorum von Cassiodor vermuten, ${ }^{32}$ aber gerade Isidor scheint eher den Ausgangspunkt der neuen mittelalterlichen Geschichtsschreibung als einen Vermittler von vorhergehenden historiografischen Erfahrungen darzustellen; und schließlich, im nun angelsächsischen Britannien, hat die querula historia von Gildas die Tradition der christlichen Invektive hinter sich, die vor allem am De gubernatione Dei des Salvianus von Marseille ${ }^{33}$ festzuhalten ist, sie zeigt aber keinen Bezug auf die antike Tradition.

Anders ist die Lage in Gallien - oder Frankreich, wenn man es so bezeichnen will. Hier hatte die heidnische Kultur dichte Spuren hinterlassen: Ausonius ist der bekannteste Fall, aber nicht der einzige, und seit kurzem wissen wir auch, dass sich Ausonius für ein eigenes, dann verloren gegangenes poetisches Werk des historischen Werkes eines gewissen Eusebius von Nantes bediente, das die gallo-römischen Usurpatoren aus der zweiten Hälfte des 3. Jahrhunderts, von Decius bis Diocletianus, behandelte $;{ }^{34}$ höchstwahrscheinlich wurde die Epitome der Historiae Philippicae des Pompeius Trogus gerade in Gallien des 4. Jahrhunderts von Justinus verfasst: $:^{35}$ Man fühlte das Bedürfnis, sie zusammenzufassen, nicht nur um eine wichtige Universalgeschichte aus der Epoche des Augustus, sondern auch das Werk einer einheimischen Persönlichkeit wie des Vokontier Pompeius Trogus zu überliefern. Sonst war die Erinnerung an Caesar, der das Land erobert und romanisiert hatte, noch lebendig, und zwar auch durch seine Schriften und die Werke seiner Biografen:

31 Über die Eigenschaften der spätantiken afrikanischen Geschichtsschreibung vgl. Zecchini (s. Anm. 1), 213-227.

32 Nicht aber eine direkte Kenntnis: so Zecchini (s. Anm. 1), 239-240.

33 So Croke (s. Anm. 7), 376.

34 Eusebius von Nantes erscheint bekanntlich in einem Katalog der Werke des Ausonius, von Giovanni de Matociis überliefert und von R. Weiss veröffentlicht, Ausonius in the fourteenth century, in: R. Bolgar (Hrsg.), Classical Influences on European Culture AD 500-1500, Cambridge 1971, 67-72; über seine Stellung innerhalb der gallorömischen Geschichtsschreibung habe ich den Forschungsstand dargestellt in G. Zecchini, Qualche ulteriore riflessione su Eusebio di Nantes e l'EKG, in: Historiae Augustae Colloquium Genevense, Bari 1999, 331-344.

35 Nach der bekannten Hypothese von Syme (s. Anm. 19). 
Die Commentarii wurden wahrscheinlich noch am Ende des 4. Jahrhunderts sogar in den Schulen gelesen (so könnte man wenigstens aus den sogenannten Hermeneúmata von Sponheim schließen), die Ephemeris von Balbus war 477 Sidonius Apollinaris noch zugänglich, ein Dichter wie Merobaudes und ein Historiker wie Frigeridus benutzten noch in der ersten Hälfte des 5. Jahrhunderts die Vita Caesaris von C. Oppius und zur gleichen Zeit war Iulius Exsuperantius dabei, Sallust zusammenzufassen. ${ }^{36}$

In den Hintergrund dieser historiografischen Lektüren ist die Blüte der spätantiken, gallo-römischen Geschichtsschreibung einzubetten, die von zwei Historiae-Schreibern vertreten wird, dem wahrscheinlich noch heidnischen Sulpicius Alexander und dem bestimmt schon christlichen Renatus Profuturus Frigeridus. Hier möchte ich nicht wieder auf die zwei Theorien eingehen, diejenige von Fr. Paschoud und meine, nach denen beide Historiker als zwei Fortsetzer des Ammianus oder als zwei Lokalhistoriker (wie meines Erachtens schon Eusebius von Nantes) zu betrachten wären $;^{37}$ ich möchte jetzt lieber hervorheben, dass die historiografische Produktion in der ersten Hälfte des 5. Jahrhunderts in Gallien ein hohes Niveau erreicht hatte (es handelt sich in beiden Fällen um Historiae in mehreren Büchern und mit zahlreichen antiken Anklängen), das in keinem anderen außer-italischen Land anzutreffen ist.

Diese außergewöhnliche Kontinuität in den historiografischen Interessen, die sich ohne Unterbrechung vom Heidentum zum Christentum, von der Epoche des Ausonius in die Zeit des Sidonius Apollinaris fortsetzt, hat nur eine einzige Entsprechung in der spätantiken Welt, und zwar den Übergang von den historíai von heidnischen Autoren wie Eunapios und Olympiodoros zu denen der christlichen Autoren wie Priskos von Panion im griechischen Orient: In beiden Fällen hatte die Dauerhaftigkeit und die tiefe Verwurzelung der Tradition einer Gattung, eben der historiografischen, die Oberhand über die sozio-kulturellen Verwandlungen zwischen Spätantike und Mittelalter, sei es barbarisch oder byzantinisch, gewonnen.

Das erklärt auch die nachfolgende Phase: Ich spreche von der Entstehung des ersten großen Werkes der mittelalterlichen Geschichtsschreibung, der zehn Libri historiarum des Gregor von Tours, die um 590 vollendet waren: $:^{38}$ Etwas

36 Vgl. Zecchini (s. Anm. 1), 154-155 und 163-179.

37 Ich habe beide Stellungnahmen zusammenfassend dargestellt in G. Zecchini, Latin Historiography: Jerome, Orosius and the Western Chronicles, in: Marasco (s. Anm. 7), 317-345, 333-335. Über Frigeridus vgl. auch P. Wynn, Frigeridus, the British Tyrants and the early fifth century barbarian invasions of Gaul and Spain, Athenaeum 85, 1998, 69-117.

38 Ich beschränke mich auf wenige bibliografische Hinweise: I. N. Wood, Gregory of Tours, Bangor 1994; M. Heinzelmann, Gregor von Tours (538-594), Darmstadt 1994; A. H. B. Breukelaar, Historiography and episcopal Authority in sixth-century 
Ähnliches, die zwölf Bücher der Historia Gothorum von Cassiodor, war vor zwei Menschenaltern in Italien verfasst worden, aber gleich danach, am Anfang des 7. Jahrhunderts, waren die ethnografischen Monografien von Isidor von Sevilla in Spanien und von Secundus von Trient in Italien wesentlich bescheidener. Die Struktur selbst von Gregors Werk weist auf eine spätantike, heidnisch-christliche Tradition hin, und die endgültige Bestätigung dieser Abstammung leiten wir daraus ab, dass Gregor sich für die historischen Ereignisse zwischen 4. und 5. Jahrhundert dreier Quellen bedient, die er ausdrücklich zitiert, und zwar Sulpicius Alexander, Orosius und Frigeridus.

Es ist schon bemerkt worden, dass Gregors Werk keinen großen Erfolg hatte: ${ }^{39}$ Trotz einer mäßigen Kontinuität in der fränkischen historiografischen Produktion, von den Chronica des Pseudo-Fredegar bis zum Liber Historiae Francorum, wurden die Libri Historiarum in der karolingischen Renaissance von den Gelehrten verschmäht; die einzige Ausnahme ist Paulus Diaconus, der sie hoch schätzt und intensiv benutzt, und der darin ein ideales Modell für seine Monografie über die Langobarden findet. ${ }^{40}$

Mit der Untersuchung der Überlieferung und des Fortlebens von historiografischen Textbausteinen antiker und daher heidnischer Herkunft in der frühmittelalterlichen Geschichtsschreibung könnte man hier aufhören. Im ostgotischen Italien des 6. Jahrhunderts, in Konstantinopel, wo sich die Exilanten bis etwa 550 aufhielten, in Gallien im 5. Jahrhundert interessiert man sich für die Geschichte und man schreibt von der Geschichte. Von diesen drei Hauptstätten über die Bibliotheken der Symmachi und der Anicii, über Jordanes und Gregor von Tours laufen und verschmelzen diese drei Richtungen in das große historische Werk des Paulus Diaconus, der gleichzeitig zum Sammelbecken und Erneuerer einer glorreichen Tradition wird.

Aber abgesehen von den historiografischen Gattungen, was verstand man unter "Geschichte“ im Frühmittelalter? Waren die Spekulation der Antike, die Überlegungen über „wie soll man Geschichte schreiben“ und „was ist Geschichte" in den folgenden Jahrhunderten noch bekannt? Die einzige sichere Antwort auf diese Frage ist im Parisinus latinus 7530 enthalten, ${ }^{41}$ einem Sammelband von 58 vorwiegend grammatikalischen Texten. Hier ist ein

Gaul, Göttingen 1994; M. Oldoni (Hrsg.), La Storia dei Franchi: I dieci libri delle storie, I-II, Napoli 2001; Croke (s. Anm. 7), 381-387.

39 M. Sot, Les Dix livres d'histoire chez les écrivains carolingiens, in: Grégoire de Tours et l'espace gaulois, Tours 1997, 319-329.

40 Capo (s. Anm. 26), 39-74.

41 Anhaltspunkt ist die Studie von L. Holtz, Le Parisinus latinus 7530, synthèse cassinienne des arts libéraux, SM 1975, 97-152. Auf den Text nr. 44 de historia (= C. Halm [Hrsg.], Rhetores latini minores, Leipzig 1863, 588) wurde von J. P. Callu, Écrire l'histoire à la fin de l'empire, in: Histoire et historiographie dans l'Antiquité, Paris 2001, 205-221 = Id. (Culture, s. Anm. 8), 7-23 hingewiesen, der ihn auch übersetzte und gebührend kommentierte. 
kurzer und anonymer Text überliefert (Nr. 44), der höchstwahrscheinlich auf den Kreis um Servius zurückgeht, also auf die antiquarische und grammatikalische Gelehrsamkeit einer noch heidnischen, spätlateinischen Epoche. ${ }^{42}$ Dieser Text enthält einige der wichtigsten Relevanzkriterien der antiken Geschichtsschreibung, die auch für die neue historiografische Kultur des Hochmittelalters gültig bleiben sollen: er besagt, dass die historia eine Erzählung der erinnerungswürdigen Ereignisse in der Kriegs- und Friedenszeit sei; der Historiker muss die Wahrheit schreiben und soll es deutlich und knapp tun; der Stil des Historikers soll elegant sein; der Zweck, das télos der Geschichte soll zweifach sein: politisch, weil sie uns lehren sollte, bestimmte Handlungen auszufuihren bzw. zu unterlassen; und kulturell, weil sie uns die Rhetorik beibringen soll. Das Vorwort eines historischen Werkes kann schließlich entweder die Geschichte an sich, oder die Persönlichkeit des Verfassers, oder das zu behandelnde Thema betreffen: Die berühmtesten Beispiele dieser drei Vorwortstypen sind Cato (die Geschichte an sich), Sallust (die Persönlichkeit des Verfassers) und Livius (das zu behandelnde Thema). Man merke den unmissverständlichen Hinweis auf die Triade der wichtigsten römischen Historiker in spätantiker Perspektive, aber auch, wenigstens was Cato und Livius anbelangt, in der Perspektive der Symmachi. ${ }^{43}$

Nun, der Parisinus latinus 7530 wurde zwischen 779 und 796 in Beneventana in Montecassino verfasst; in jenen Jahren war Paulus Diaconus der angesehenste Gast in Montecassino: Wie schon bemerkt wurde, ${ }^{44}$ ist er nicht der Autor der Sammlung, trug aber vielleicht dazu bei, indem er einige Texte lieferte oder empfahl; auf jeden Fall beeinflusste seine Persönlichkeit in entscheidender Weise den Verfasser des Sammelbandes. Paulus Diaconus erweist sich also wiederum als die zentrale Gestalt in meiner Untersuchung über das Fortleben der antiken Geschichtsschreibung, ihrer Gattungen und ihrer Ziele durch das frühe Mittelalter und bis zur karolingischen Renaissance.

Zum Abschluss möchte ich einige Betrachtungen anhängen:

Die Fach- oder pseudo-historische Literatur ist ein weiteres Mittel zur Überlieferung heidnischer Materialien in christlicher Epoche. Vier Beispiele werden ausreichen.

1) Neben der christlichen Tradition von Iulius Honorius und vor allem von Orosius, der seinerseits aus den Commentarii des Agrippa schöpft, ${ }^{45}$ und neben der Tradition der ostgotischen Geografen, die im Anonymus von

42 Über die historische Bildung des Servius vgl. jetzt A. Pellizzari, Servio: storia, cultura e istituzioni nell'opera di un grammatico tardoantico, Firenze 2003.

43 Über das spätantike Nachleben der drei Historiker vgl. Zecchini (s. Anm. 1), 150-151 und $155-157$.

44 Aus Holtz (s. Anm. 41), 142-143.

45 Vgl. Zecchini (s. Anm. 1), 258-261. 
Ravenna zitiert werden, ${ }^{46}$ wird die antike Tradition im Bereich der Landeskunde insbesondere durch folgende Autoren vertreten: a) Pomponius Mela, der sowohl dem Cassiodor als auch dem Jordanes bekannt war und der ebenfalls zu Theoderichs Zeit in Ravenna von Domnulus abgeschrieben wurde $;{ }^{47}$ b) Iulius Titianus, einen wahrscheinlich gallo-römischen Autor aus dem 3. Jahrhundert, da er dem Gregor von Tours noch zugänglich war; ${ }^{48} \mathrm{c}$ ) den geheimnisvollen Castorius, wenn auch nur hypothetisch, der ganze $36 \mathrm{mal}$ vom Anonymus von Ravenna erwähnt wird $;^{49}$ d) die lateinische Fassung der Expositio totius mundi et gentium, eines heidnischen und orientalischen, um 359 datierbaren Werkes: Seine Übersetzung ins Lateinische wurde wahrscheinlich in der ostgotischen Zeit gemacht. ${ }^{50}$

2) Im militärischen Bereich ist Vegetius bestimmt schon christlich, hat aber eine jahrhundertelange Tradition hinter sich, an der er treu festhält, ohne dass sein Glaube ihn bei der Verfassung seiner Epitoma rei militaris beeinflussen würde. Die Tatsache, dass Freculf von Lisieux den zweiten Teil seiner Historia

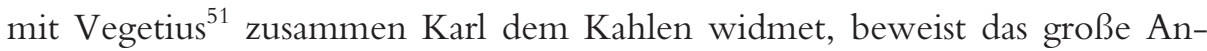
sehen des Vegetius und das andauernde Interesse der karolingischen Zeit für die antike Literatur über das Militärwesen.

3) Im pädagogischen Bereich vertritt ein um 390 im Kreis um Symmachus verfasster, also noch heidnischer Text wie die Institutio Traiani die edle Tradition der specula principum hellenistischer Abstammung, die durch eine Sektion über militärische Kunststücke bereichert wurde: Diese institutio, die sich anscheinend auf Traianus bezieht, sich aber in Wirklichkeit an Theodosius als

46 Aristarchus (?), Menelac, Anarid, Aithanarit, Eldebald und Marcomir; über gotische Geografen in Ravenna vgl. Pecere (s. Anm. 16), 384, sowie auch Heather (s. Anm. 7), 334.

47 Das entnimmt man der Unterschrift zum 3. Buch der Chorographia: Martindale (s. Anm. 15), 374-375. Über Rezeption und Überlieferung des Pomponius Mela vgl. G. Billanovich, Dall'antica Ravenna alle Biblioteche umanistiche, Aevum 30, 1956, 319354.

48 Greg. Turon. De cursu stellarum XXX; vgl. Zecchini (s. Anm. 1), 236.

49 Zecchini (s. Anm. 1), 257. Zwischen dem 4. und dem 5. Jahrhundert lebte vermutlich auch Aethicus Istros, der anonyme Verfasser einer Cosmographia zwischen Landeskunde und Thaumasiografie, die dann während des 8. Jahrhunderts von einem Presbyter namens Hieronymus auszugsweise abgeschrieben wurde: vgl. Zecchini (s. Anm. 1), 264-265, und, insbesondere, V. Peri, La Cosmographia dell'Anonimo di Histria e il suo compendio dell'VIII secolo, in: R. Avesani (Hrsg.), Vestigia. Studi in onore di Giuseppe Billanovich, Roma 1984, II, 503-558; jetzt aber auch O. Prinz (Hrsg.), Die Kosmographie des Aethicus, München 1993, der die Entstehung des ganzen Werkes auf das 8. Jahrhundert zurückdatiert - ich weiß nicht, ob mit Recht.

50 So lautet wenigstens die bekannte Hypothese von J. Rougé (Hrsg.), Expositio totius mundi et gentium, Paris 1966, 101-102.

51 So Chiesa (s. Anm. 21), 245. Allgemein über Vegetius vgl. M. D. Reeve, The Transmission of Vegetius's Epitoma rei militaris, Aevum 74, 2000, 244-354. 
den neuen Traianus wendet, gelangte bis zu John of Salisbury, der lange Auszüge davon abschrieb: Zweifellos wurde ihr Überleben durch das frühe Mittelalter hindurch von der weitverbreiteten Legende des christlichen Glaubens von Traianus selbst begünstigt. ${ }^{52}$

4) Erzählerische Texte, die aber eine historische Grundlage haben oder die man als historisch betrachtete wie die Historia Apollonii regis Tyrii, die De excidio Troiae historia von Dares aus Phrygien und das corpus der Texte, die Alexander betrafen, erhalten sich problemlos bis ins frühe Mittelalter hinein, indem sie nicht rein historische Bedürfnisse befriedigen, aber die Erinnerung an Ereignisse lebendig halten, die weit zurück in der Vergangenheit liegen (der Trojanische Krieg, Alexander der Große) und die man in den bekanntesten Universalgeschichten wiederfinden kann, d. h. bei Orosius und Justinus. Insbesondere überlebt die antike historiografische Tradition über Alexander den Großen in gut drei Rezeptionslinien: in der von Justinus ,über Pompeius Trogus" überlieferten, hellenistischen Linie, in der griechisch-kaiserlichen Linie des Arrianus, die im Itinerarium Alexandri wiederzufinden ist, und in der lateinisch-kaiserlichen Linie des Curtius Rufus, dessen Spuren in der Epitoma rerum gestarum Alexandri und im Liber de morte Alexandri Magni testamentoque eius erhalten sind, der übrigens ihre frühzeitige Christianisierung bezeugt. ${ }^{53}$

52 Ich fasse hier kurz zusammen, was in Zecchini (s. Anm. 1), 53-54 und 137 ausführlich dargestellt wurde.

53 Vgl. L. Cracco Ruggini, L'Epitoma Rerum Gestarum Alexandri Magni e il Liber de Morte testamentoque eius, Athenaeum 39, 1961, 285-357; Ead., Sulla cristianizzazione della cultura pagana: il mito greco e latino di Alessandro dall'età antonina al Medioevo, Athenaeum 43, 1965, 3-39; J. P. Callu, Alexandre dans la littérature latine de l'Antiquité tardive, in: Alexandre le Grand dans les littératures occidentales et proche-orientales, Paris 1999, 33-50 = Id. (Culture, s. Anm. 8), 565-582. Die Historia de preliis, d.h. die lateinische Übersetzung des Alexanderromans des PseudoKallisthenes, verstärkte weiterhin die mittelalterliche Alexander-Historiografie; sie wurde aber erst um 950 in Neapel von Leo Diaconus zu Ende gebracht (vgl. Chiesa [s. Anm. 21], 252-253). 
